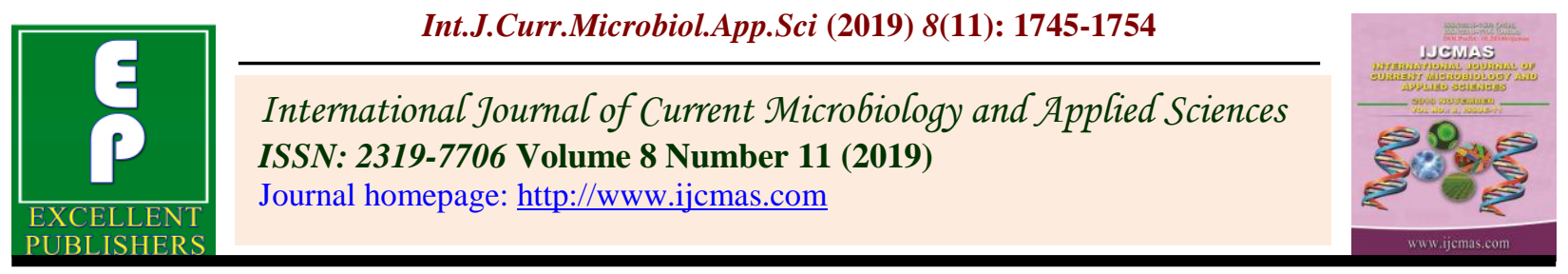

Original Research Article

https://doi.org/10.20546/ijcmas.2019.811.204

\title{
Effect of Delayed Harvesting and Crushing on Yield and Juice Quality of Promising Sugarcane Clones
}

\author{
B. Vajantha*, M. Hemanth Kumar, K.R. Tagore, N.V. Sarala and T.M. Hemalatha
}

Agricultural Research Station, Perumallapalle, Tirupati-517 505, Andhra Pradesh, India

*Corresponding author

\section{A B S T R A C T}

\section{Keywords}

Sugarcane clones, Delayed harvesting and crushing, Cane weight and Juice quality

Article Info

Accepted:

17 October 2019

Available Online:

10 November 2019
The most important and dominating fact in pulling down sugar recovery is delayed harvesting and crushing of cane. The deterioration in cane weight and juice quality due to late harvest and delayed crushing are especially important in Andhra Pradesh where the price of cane is paid on weight basis. In this view the study was undertaken to assess the extent of loss in cane weight and quality of juice in various sugarcane clones with delayed harvest and delayed crushing during 2018-19 at Agricultural Research Station, Perumallapalle, Tirupati, Andhra Pradesh. The change in cane weight and juice quality parameters viz., sucrose percent and commercial cane sugar (CCS) percent were recorded at each month of harvesting and

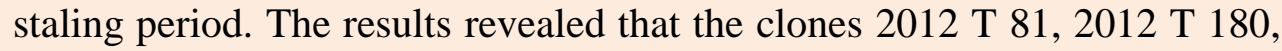
2012 T 72, 2012 T 182 and 2012 T 78 were suitable for delayed harvesting. However, 2012 T 81 and 2012 T 182 are showed tolerance to post harvest deterioration when compared with other clones.

\section{Introduction}

Sugarcane is the perishable commodity and must be processed into sugar quickly after it is harvested. Post harvest sucrose lasses have been reported from many canes producing countries and linked with low sugar recovery several problems during sugar processing. Studies have indicated that nearly $20-30 \%$ of total sucrose synthesized by sugarcane plant is lost during various stages of raw material handling and sugar mill processing. In subtropical India, cane starts ripening from December onwards. Besides external factors, the intrinsic factors such as genetic and biochemical constitution of genotypes, stage of maturity and cane quality at harvest affect the rate of post harvest deterioration of canes.

Delayed harvesting or harvesting of over aged canes leads to deterioration of canes. Over aged canes will have more fiber, less Juice, more dead and dry canes, more pith and thus will affect recovery. Optimum age for early 
varieties should not be less than 10 months and for midlate varieties it is 12 months. However some early clones reach peak maturity at around 11 months and if they are delayed very much, pith formation and reduction in cane weight and quality will be occurs. Nearly one fourth of crushed cane in Indian Sugar Factories has been found to be stale in quality (Sharma and Sunitha, 1994). The loss is increases with the increase in duration of staling and variation with varieties (Solomon et al., 1997).

The sugarcane yield has been increased over the years due to release of high yielding clones and due to agro management practices. However the sugar recovery is stable and which mainly depends on cane quality, efficiency of mills, planting and harvesting dates as well as staling period. Sugarcane varieties play a crucial role in sugar recovery, depending upon the climate and management practices followed. A very big difference in susceptibility to post harvest deterioration has been noticed which is important in countries where there are prolonged harvest to milling delay. Keeping this in view the present investigation was carried out to evaluate the promising sugarcane clones for delayed harvesting and post harvest deterioration.

\section{Materials and Methods}

The experiment was carried out to study the impact of delayed harvesting and delayed crushing on can yield and quality of sugarcane clones at Agricultural Research Station, Perumallapalle, Tirupati, ANGRAU during 2018-19. Eight promising sugarcane clones along with two check varieties were evaluated. The field experiment was laid out in split split plot design with three replications. The experiment consists of main treatments $(10: 8$ clones +2 check varieties), sub treatments (Months of harvest - early clones were harvested at 10, 11, 12 months after planting and Midlate clones were harvested at 12, 13, 14 months after planting) and sub sub treatments (Staling period - Juice analysis was done at $1^{\text {st }}, 2^{\text {nd }}, 3^{\text {rd }}, 4^{\text {th }}$ and $5^{\text {th }}$ day after harvesting). The selected clones were planted with a seed rate of 40,000 three budded setts/ ha. Atrazine @ $2 \mathrm{~kg}$ a.1/ha was sprayed as pre emergence herbicide at $2^{\text {nd }}$ day after planting. Fertilizer dose of 224-112-112 Kg N-P $\mathrm{O}_{5^{-}}$ $\mathrm{K}_{2} \mathrm{O}$ was followed. Other cultural operations like hand weeding, earthing up, trash twist propping etc., were practiced as per recommendations. The data on yield and juice quality parameters viz., sucrose percent and CCS percent were recorded at each month of harvesting and staling period of cane from $1^{\text {st }}$ day to $5^{\text {th }}$ day. Total soluble solids were measured by using Brix hydrometer with the method given by Meade and Chen (1977) and the reading was corrected to temperature by referring the tables. Sucrose was analyzed in cane juice and expressed in percent. The data was statistically analyzed by the method outlined by Panse and Sukhatme (1085) by using SPSS 20.

\section{Results and Discussion}

\section{Cane weight}

Cane weight was significantly affected by varieties, months of harvest and staling period. However the interaction between varieties, months of harvest and staling period was non significant (Table 1).

\section{Effect of delayed harvesting on cane weight}

Cane weight was significantly affected by varieties, months as well as their interaction. Among early clones, cane weight was increased from 10 to 11 months in $2012 \mathrm{~T} 81$ and $2012 \mathrm{~T}$ 106. The highest increase (7.10\%) in cane weight from 10 to 11 months was recorded in $2012 \mathrm{~T} 81$ followed by $2012 \mathrm{~T}$ $106(0.40 \%)$. Remaining early clones showed 
decreased trend in cane weight from 10 to 11 months. All the early clones showed decrease in cane weight from 10 to 12 months. $2012 \mathrm{~T}$ 81 recorded less reduction in cane weight ($1.28 \%)$ followed by $2012 \mathrm{~T} 180$ $(-4.7 \%)$. When compared to check variety 2003V46, 2012T73 registered more reduction in cane weight from 10 to 11 months and 10 to 12 months. Among midlate clones, 2012T78 and 2012 T 182 showed increase in cane weight from 12 to 13 months $(2.9 \%$ and $0.96 \%$, respectively) and from 12 to 14 months $(0.7 \%$ and $0.92 \%$, respectively) (Table 4).

\section{Effect of delayed crushing on cane weight}

Varieties and staling period showed significant effect on cane weight however the interaction effect was not significant.

The cane weight was decreased with increasing staling period. Among early cones, the check variety 2003 V 46 showed less reduction in mean cane weight from $1^{\text {st }}$ day to $5^{\text {th }}$ day $(-4.43 \%)$ followed by $2012 \mathrm{~T} 81$ ($5.93 \%)$. In midlate clones, $2012 \mathrm{~T} 72$ recorded less reduction in mean cane weight $(-3.49 \%)$ followed by $2012 \mathrm{~T} 182(-3.74 \%)$.

All the clones showed decreasing trend in cane weight with increasing staling period from $1^{\text {st }}$ day to $5^{\text {th }}$ day. Cane weight loss is mainly attributed to evaporation loss and respiratory losses (Alexander, 1973).The results were in concurrence with the findings of Siddhant et al., (2008) (Table 4).

\section{Sucrose percent}

The data in table 2 indicated that varieties, months of harvest and staling period showed significant influence on sucrose percent. However the interaction between varieties, months of harvest and staling period was non significant (Table 2).

\section{Effect of delayed harvesting on sucrose}

The sucrose content was significantly effected by varieties, months as well as with their interaction. Among the early clones $2012 \mathrm{~T}$ 180 showed less reduction in sucrose content from 10 to 11 months $(-0.8 \%)$ and 10 to 12 months $(-0.4 \%)$, followed by $2012 \mathrm{~T} 73$ (from 10 to 11 months) and $2012 \mathrm{~T} 106$ (10 to 12 months). The midlate clone 2012 T 182 recorded highest increase in sucrose percent (4.6\%) from 12 to 13 months harvesting. However the clone 2012 T 72 showed less reduction in sucrose percent $(-2.4 \%)$ from 12 to 14 months followed by $2012 \mathrm{~T} 58(-3.9 \%)$.

\section{Effect of delayed crushing on sucrose}

The sucrose content was significantly influenced by varieties and staling period. The interaction effect between varieties and days was non significant (Table 5). Among the five early clones, 2012 T 81 showed less reduction in mean sucrose from $1^{\text {st }}$ day to $5^{\text {th }}$ day at all months of harvesting. $6.40 \%$ and $0.57 \%$ reduction in sucrose was noticed at $10^{\text {th }}$ month and $11^{\text {th }}$ month harvest, respectively. The less mean reduction in sucrose percent was observed with $2012 \mathrm{~T}$ 81(-2.90\%) followed by 2012 T 73 (-4.26\%). When compared check variety 2003 V 46, all early clones showed less reduction in mean sucrose content. Among the midlate clones 2012 T 182 showed less reduction in mean sucrose from $1^{\text {st }}$ day to $5^{\text {th }}$ day $(-9.55 \%)$. During storage, inversion of sucrose starts resulting in the formation of invert sugar which leads to loss of recoverable sugar (Gupta et al., 1975) (Table 6).

\section{Commercial Cane Sugar (CCS)}

The varieties, months of harvest and staling period showed significant influence on CCS percent. However the interaction between varieties, months of harvest and staling period was non significant (Table 3). 
Table.1 Effect of delayed harvesting and crushing on cane weight ( $\mathrm{kg} /$ single cane) of promising sugarcane clones

\begin{tabular}{|c|c|c|c|c|c|c|c|c|c|c|c|c|c|c|c|c|c|c|}
\hline Entries & 1 day & 2 day & 3 day & 4 day & 5 day & Mean & 1 day & 2 day & 3 day & 4 day & 5 day & Mean & 1 day & 2 day & 3 day & 4 day & 5 day & Mean \\
\hline Early & \multicolumn{6}{|c|}{$10^{\text {th }}$ month } & \multicolumn{6}{|c|}{$11^{\text {th }}$ month } & \multicolumn{6}{|c|}{$12^{\text {th }}$ month } \\
\hline $2012 \mathrm{~T}$ & 1.747 & 1.704 & 1.664 & 1.628 & 1.607 & 1.670 & 1.573 & 1.528 & 1.500 & 1.479 & 1.468 & 1.509 & 1.464 & 1.438 & 1.416 & 1.338 & 1.356 & 1.403 \\
\hline $2012 T$ & 1.658 & 1.598 & 1.582 & 1.569 & 1.556 & 1.593 & 1.651 & 1.617 & 1.597 & 1.574 & 1.555 & 1.599 & 1.554 & 1.529 & 1.507 & 1.490 & 1.463 & 1.509 \\
\hline $2012 \mathrm{~T}$ & 1.544 & 1.503 & 1.473 & 1.454 & 1.450 & 1.485 & 1.630 & 1.612 & 1.599 & 1.579 & 1.569 & 1.598 & 1.234 & 1.219 & 1.158 & 1.126 & 1.092 & 1.166 \\
\hline $2012 \mathrm{~T}$ & 1.517 & 1.483 & 1.456 & 1.437 & 1.411 & 1.461 & 1.472 & 1.442 & 1.423 & 1.409 & 1.394 & 1.428 & 1.434 & 1.423 & 1.404 & 1.369 & 1.348 & 1.396 \\
\hline $2003 \mathrm{~V}$ & 1.726 & 1.693 & 1.672 & 1.653 & 1.645 & 1.678 & 1.653 & 1.629 & 1.609 & 1.591 & 1.580 & 1.612 & 1.445 & 1.422 & 1.411 & 1.394 & 1.384 & 1.411 \\
\hline Midlate & \multicolumn{6}{|c|}{$12^{\text {th }}$ month } & \multicolumn{6}{|c|}{$13^{\text {th }}$ month } & \multicolumn{6}{|c|}{$14^{\text {th }}$ month } \\
\hline $2012 \mathrm{~T}$ & 1.812 & 1.768 & 1.745 & 1.729 & 1.712 & 1.753 & 1.513 & 1.502 & 1.494 & 1.481 & 1.472 & 1.492 & 1.442 & 1.431 & 1.414 & 1.404 & 1.389 & 1.416 \\
\hline $2012 \mathrm{~T}$ & 1.417 & 1.403 & 1.392 & 1.377 & 1.363 & 1.390 & 1.410 & 1.394 & 1.380 & 1.369 & 1.361 & 1.383 & 1.356 & 1.347 & 1.334 & 1.321 & 1.313 & 1.334 \\
\hline $2012 \mathrm{~T}$ & 1.414 & 1.378 & 1.375 & 1.349 & 1.332 & 1.369 & 1.419 & 1.402 & 1.390 & 1.378 & 2.368 & 1.591 & 1.397 & 1.393 & 1.382 & 1.367 & 1.357 & 1.379 \\
\hline $2012 \mathrm{~T}$ & 1.130 & 1.118 & 1.106 & 1.099 & 1.093 & 1.109 & 1.257 & 1.245 & 1.231 & 1.222 & 1.211 & 1.233 & 1.148 & 1.133 & 1.117 & 1.102 & 1.099 & 1.120 \\
\hline Co & 1.206 & 1.178 & 1.158 & 1.114 & 1.090 & 1.149 & 1.241 & 1.225 & 1.209 & 1.197 & 1.182 & 1.211 & 1.212 & 1.199 & 1.183 & 1.176 & 1.155 & 1.185 \\
\hline
\end{tabular}

\begin{tabular}{|c|c|c|}
\hline Treatments & Sig & p value \\
\hline Varieties & $* *$ & 0.000 \\
\hline Months & $* *$ & 0.000 \\
\hline Varieties * Months & $*$ & 0.037 \\
\hline Staling period & $* *$ & 0.000 \\
\hline Varieties * Staling period & N.S. & 0.995 \\
\hline Months * Staling period & N.S. & 0.066 \\
\hline Varieties * Months $*$ Staling & N.S. & 0.830 \\
\hline
\end{tabular}


Table.2 Effect of delayed harvesting and crushing on sucrose (\%) of promising sugarcane clones

\begin{tabular}{|c|c|c|c|c|c|c|c|c|c|c|c|c|c|c|c|c|c|c|}
\hline Entries & 1 day & 2 day & 3 day & 4 day & 5 day & Mean & 1 day & 2 day & 3 day & 4 day & 5 day & Mean & 1 day & 2 day & 3 day & 4 day & 5 day & Mean \\
\hline Early & \multicolumn{6}{|c|}{$10^{\text {th }}$ month } & \multicolumn{6}{|c|}{$11^{\text {th }}$ month } & \multicolumn{6}{|c|}{$12^{\text {th }}$ month } \\
\hline 2012 T 73 & 18.46 & 18.42 & 17.56 & 17.23 & 16.94 & 17.72 & 17.75 & 18.13 & 17.17 & 17.59 & 17.02 & 17.53 & 16.58 & 17.8 & 19.07 & 16.79 & 16.62 & 17.37 \\
\hline 2012 T 81 & 17.91 & 17.66 & 17.52 & 17.44 & 16.76 & 17.46 & 16.5 & 17.22 & 16.21 & 16.15 & 16.91 & 16.6 & 16.08 & 15.39 & 14.38 & 16.38 & 15.8 & 15.61 \\
\hline 2012 T 106 & 18.11 & 17.98 & 17.8 & 16.54 & 16.4 & 17.36 & 17.48 & 17.63 & 17.26 & 16.25 & 16.82 & 17.09 & 17.75 & 17.26 & 17.22 & 16.82 & 16.56 & 17.12 \\
\hline 2012 T 180 & 17.71 & 16.7 & 16.56 & 16.43 & 16.34 & 16.75 & 17.7 & 17.27 & 16.41 & 16.01 & 15.7 & 16.62 & 16.36 & 16.23 & 16.77 & 16.8 & 17.26 & 16.68 \\
\hline 2003 V 46 & 19.22 & 18.91 & 18.66 & 17.81 & 17.11 & 18.34 & 18.04 & 18.03 & 17.67 & 16.35 & 17.31 & 17.48 & 17.47 & 17.25 & 17.12 & 16.31 & 15.84 & 16.8 \\
\hline Midlate & \multicolumn{6}{|c|}{$12^{\text {th }}$ month } & \multicolumn{6}{|c|}{$13^{\text {th }}$ month } & \multicolumn{6}{|c|}{$14^{\text {th }}$ month } \\
\hline 2012 T 58 & 18.51 & 18.12 & 17.96 & 18.07 & 15.33 & 17.62 & 18.22 & 17.94 & 17.87 & 17.73 & 17.35 & 17.82 & 18.36 & 17.25 & 16.91 & 16.83 & 15.34 & 16.94 \\
\hline 2012 T 72 & 17.36 & 17.12 & 16.96 & 16.82 & 14.43 & 16.72 & 18.08 & 18.24 & 16.51 & 16.47 & 16.67 & 17.2 & 17.38 & 16.83 & 16.54 & 15.96 & 14.43 & 16.32 \\
\hline 2012 T 78 & 18.41 & 17.93 & 17.66 & 17.6 & 17.12 & 17.74 & 18.53 & 17.24 & 16.71 & 16.37 & 16.18 & 17.01 & 19.03 & 17.2 & 16.31 & 16.24 & 16.15 & 16.98 \\
\hline 2012 T 182 & 16.37 & 17.52 & 17.42 & 16.99 & 15.15 & 16.7 & 18.18 & 17.93 & 17.83 & 17.4 & 15.95 & 17.46 & 15.89 & 15.3 & 15.13 & 15.16 & 14.47 & 15.19 \\
\hline Co 99004 & 19.06 & 18.58 & 18.41 & 18.08 & 17.69 & 18.36 & 19.85 & 17.79 & 17.31 & 16.71 & 16.33 & 17.6 & 19.06 & 18.6 & 18.42 & 18.08 & 17.7 & 18.37 \\
\hline
\end{tabular}

\begin{tabular}{|c|c|c|}
\hline Treatments & Sig & p value \\
\hline Varieties & $* *$ & 0.000 \\
\hline Months & $* *$ & 0.006 \\
\hline Varieties * Months & $* *$ & 0.000 \\
\hline Staling period & $* *$ & 0.000 \\
\hline Varieties * Staling period & N.S. & 0.905 \\
\hline Months * Staling period & N.S. & 0.910 \\
\hline Varieties * Months * Staling & N.S. & 1.000 \\
\hline
\end{tabular}


Table.3 Effect of delayed harvesting and crushing on CCS (\%) of promising sugarcane clones

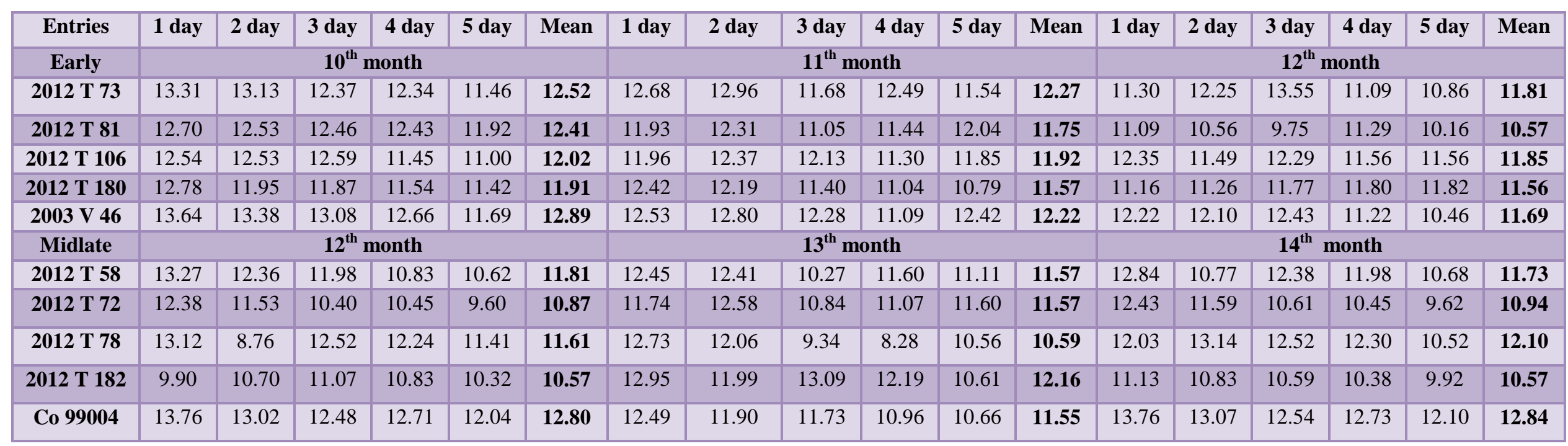

\begin{tabular}{|c|c|c|}
\hline Treatments & Sig & p value \\
\hline Varieties & $* *$ & 0.000 \\
\hline Months & $*$ & 0.050 \\
\hline Varieties * Months & $* *$ & 0.000 \\
\hline Staling period & $* *$ & 0.000 \\
\hline Varieties * Staling period & N.S. & 0.977 \\
\hline Months * Staling period & N.S. & 0.205 \\
\hline Varieties $*$ Months * Staling period & N.S. & 0.674 \\
\hline
\end{tabular}


Table.4 Effect of delayed harvesting on cane weight of promising sugarcane clones

\begin{tabular}{|c|c|c|c|c|c|}
\hline \multirow{3}{*}{$\begin{array}{c}\text { Entries } \\
\text { Early }\end{array}$} & \multicolumn{3}{|c|}{ Mean cane weight ( $\mathrm{kg}$ / single cane) } & \multirow{2}{*}{\multicolumn{2}{|c|}{$\begin{array}{c}\% \text { increase / decrease in cane } \\
\text { weight }\end{array}$}} \\
\hline & \multicolumn{3}{|c|}{ Harvested at } & & \\
\hline & $10^{\text {th }}$ month & $11^{\text {th }}$ month & $12^{\text {th }}$ month & 10 to 11 months & 10 to 12 months \\
\hline 2012 T 73 & 1.670 & 1.509 & 1.403 & -10.6 & -19.1 \\
\hline 2012 T 81 & 1.485 & 1.598 & 1.466 & 7.10 & -1.28 \\
\hline 2012 T 106 & 1.593 & 1.599 & 1.509 & 0.40 & -5.60 \\
\hline 2012 T 180 & 1.461 & 1.428 & 1.396 & -2.3 & -4.7 \\
\hline 2003 V 46 (C) & 1.678 & 1.612 & 1.411 & -4.1 & -18.9 \\
\hline Midlate & $12^{\text {th }}$ month & $13^{\text {th }}$ months & $14^{\text {th }}$ months & 12 to 13 months & 12 to 14 months \\
\hline 2012 T 58 & 1.753 & 1.620 & 1.516 & -8.2 & -15.7 \\
\hline 2012 T 72 & 1.390 & 1.383 & 1.334 & -0.5 & -4.2 \\
\hline 2012 T 78 & 1.369 & 1.410 & 1.379 & 2.9 & 0.7 \\
\hline 2012 T 182 & 1.109 & 1.133 & 1.120 & 0.96 & 0.92 \\
\hline CO 99004 & 1.149 & 1.211 & 1.185 & 5.40 & 3.13 \\
\hline & & Varieties & Months & Varieties*Mol & \\
\hline & Sig & & & & \\
\hline & p value & 0.0 & & 0. & \\
\hline
\end{tabular}

Table.5 Percent reduction in cane weight and sucrose of promising sugarcane clones from $1^{\text {st }}$ day to $5^{\text {th }}$ day

\begin{tabular}{|c|c|c|c|c|c|c|c|c|}
\hline Entries & \multicolumn{3}{|c|}{ Cane weight } & & \multicolumn{3}{|c|}{ Sucrose } & \\
\hline Early & $\begin{array}{l}1^{\text {th }} \\
\text { month }\end{array}$ & $\begin{array}{l}11^{\text {th }} \\
\text { month }\end{array}$ & $\begin{array}{l}12^{\text {th }} \\
\text { month }\end{array}$ & Mean & $\begin{array}{c}1^{\text {th }} \\
\text { month }\end{array}$ & $\begin{array}{l}11^{\text {th }} \\
\text { month }\end{array}$ & $\begin{array}{l}12^{\text {th }} \\
\text { month }\end{array}$ & Measn \\
\hline 2012 T 73 & -8.05 & -6.66 & -7.33 & -7.35 & -8.24 & -4.15 & -0.38 & -4.26 \\
\hline 2012 T 81 & -6.11 & -5.81 & -5.85 & -5.93 & -6.40 & -0.57 & -1.74 & -2.90 \\
\hline 2012 T 106 & -6.08 & -3.70 & -11.54 & -7.11 & -9.44 & -3.81 & -6.69 & -6.65 \\
\hline 2012 T 180 & -6.93 & -5.30 & -6.04 & -6.09 & -7.72 & -11.30 & -0.59 & -6.54 \\
\hline 2003 V 46 @ & -4.66 & -4.44 & -4.18 & -4.43 & -10.96 & -4.05 & -9.33 & -8.11 \\
\hline Midlate & $\begin{array}{l}12^{\text {th }} \\
\text { month }\end{array}$ & $\begin{array}{l}13^{\text {th }} \\
\text { month }\end{array}$ & $\begin{array}{c}14^{\text {th }} \\
\text { month }\end{array}$ & Mean & $\begin{array}{l}12^{\text {th }} \\
\text { month }\end{array}$ & $\begin{array}{l}13^{\text {th }} \\
\text { month }\end{array}$ & $\begin{array}{l}14^{\text {th }} \\
\text { month }\end{array}$ & Mean \\
\hline 2012 T 58 & -5.52 & -2.71 & -3.63 & -3.95 & -11.76 & -4.76 & -16.45 & -10.99 \\
\hline 2012 T 72 & -3.81 & -3.47 & -3.19 & -3.49 & -16.89 & -7.80 & -16.94 & -13.88 \\
\hline 2012 T 78 & -5.78 & -3.57 & -2.91 & -4.09 & -7.03 & -12.67 & -15.13 & -11.61 \\
\hline 2012 T 182 & -3.26 & -3.71 & -4.24 & -3.74 & -7.43 & -12.28 & -8.93 & -9.55 \\
\hline CO 99004 & -8.81 & -4.75 & -4.70 & -6.11 & -7.18 & -17.73 & -10.28 & -11.73 \\
\hline
\end{tabular}


Table.6 Effect of delayed harvesting on sucrose of promising sugarcane clones

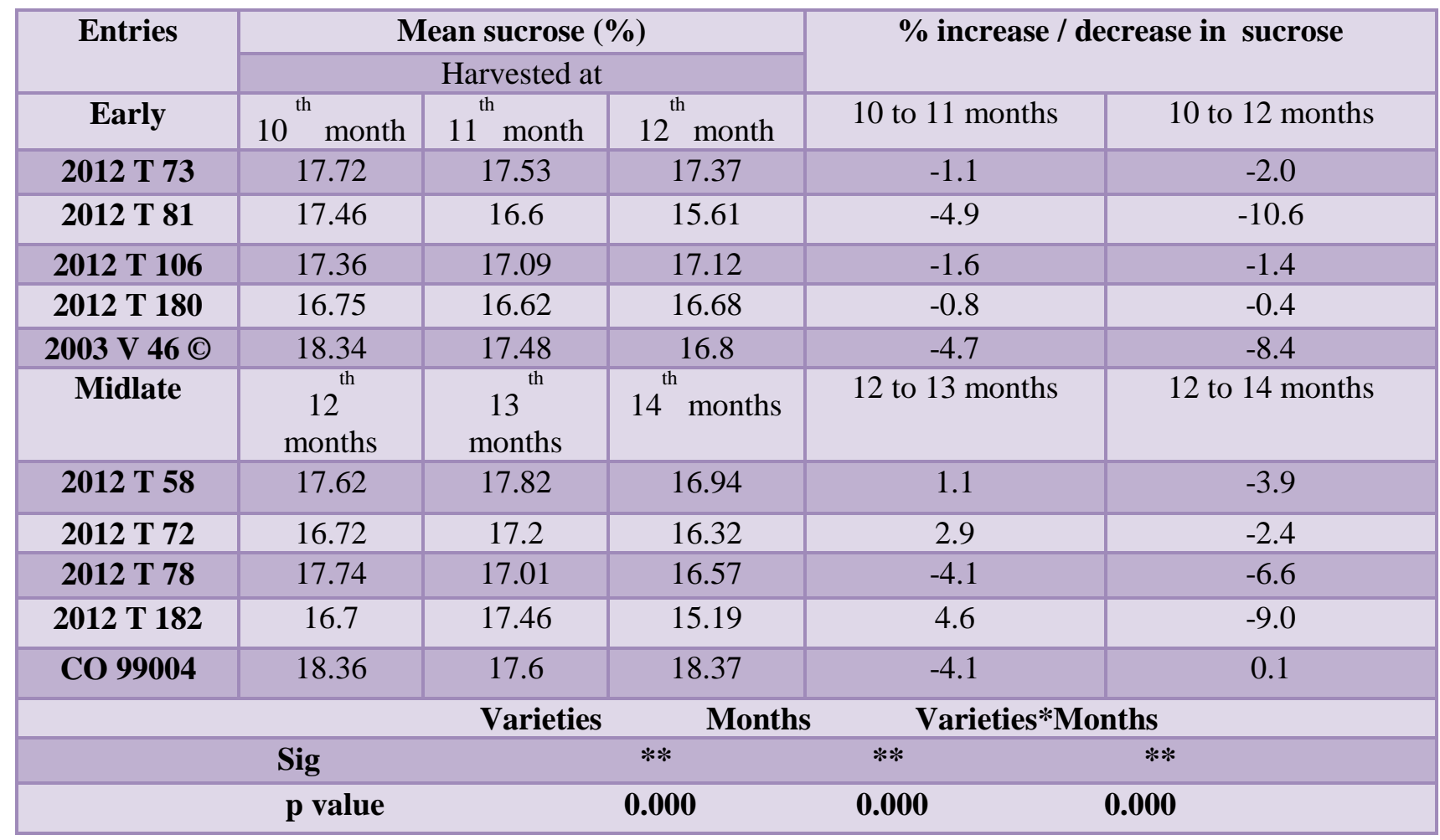

Table.7 Effect of delayed harvesting on CCS \% of promising sugarcane clones

\begin{tabular}{|c|c|c|c|c|c|}
\hline \multirow{3}{*}{$\begin{array}{c}\text { Entries } \\
\text { Early }\end{array}$} & \multirow{2}{*}{\multicolumn{3}{|c|}{$\begin{array}{c}\text { Mean CCS (\%) } \\
\text { Harvested at }\end{array}$}} & \multirow{2}{*}{\multicolumn{2}{|c|}{$\%$ increase / decrease in CCS }} \\
\hline & & & & & \\
\hline & \multicolumn{2}{|c|}{ th $\quad$ Harvested a } & $12^{\text {th }}$ month & 10 to 11 months & 10 to 12 months \\
\hline 2012 T 73 & 12.52 & 12.27 & 11.81 & -2.00 & -5.67 \\
\hline 2012 T 81 & 12.41 & 11.75 & 10.57 & -5.32 & -14.83 \\
\hline 2012 T 106 & 12.02 & 11.92 & 11.85 & -0.83 & -1.41 \\
\hline 2012 T 180 & 11.91 & 11.57 & 11.56 & -2.85 & -2.94 \\
\hline $2003 \mathrm{~V} 46$ C & 12.89 & 12.22 & 11.69 & -5.20 & -9.31 \\
\hline Midlate & $\begin{array}{l}12^{\text {th }} \\
\text { months }\end{array}$ & $\begin{array}{l}13^{\text {th }} \\
\text { months }\end{array}$ & $14^{\text {th }}$ months & 12 to 13 months & 12 to 14 months \\
\hline 2012 T 58 & 11.81 & 11.57 & 11.73 & -2.03 & -0.68 \\
\hline 2012 T 72 & 10.87 & 11.57 & 10.94 & 6.44 & 0.64 \\
\hline 2012 T 78 & 11.61 & 10.59 & 12.1 & -8.79 & 4.22 \\
\hline 2012 T 182 & 10.57 & 12.16 & 10.57 & 15.04 & -0.00 \\
\hline CO 99004 & 12.8 & 11.55 & 12.84 & -9.77 & 0.31 \\
\hline & & \multicolumn{2}{|c|}{ Varieties } & \multicolumn{2}{|c|}{ Varieties*Months } \\
\hline \multicolumn{3}{|c|}{ Sig } & $* *$ & \multicolumn{2}{|c|}{$* *$} \\
\hline \multicolumn{3}{|c|}{ p value } & 0.000 & \multicolumn{2}{|c|}{0.000} \\
\hline
\end{tabular}


Table.8 Percent reduction in CCS of promising sugarcane clones from $1^{\text {st }}$ day to $5^{\text {th }}$ day

\begin{tabular}{|c|c|c|c|c|c|c|c|c|c|c|}
\hline \multirow{3}{*}{$\begin{array}{l}\text { Entries } \\
\text { Early }\end{array}$} & \multicolumn{6}{|c|}{$\operatorname{CCS} \%$} & \multicolumn{4}{|c|}{$\%$ decreas in CCS \% } \\
\hline & \multicolumn{2}{|c|}{$\begin{array}{c}{ }^{\text {th }} \\
10^{2} \\
\text { month }\end{array}$} & \multicolumn{2}{|c|}{$\begin{array}{l}11^{\text {th }} \\
\text { month }\end{array}$} & \multicolumn{2}{|c|}{$\begin{array}{l}12^{\text {th }} \\
\text { month }\end{array}$} & \multirow[t]{2}{*}{$\begin{array}{l}\text { th } \\
10^{\text {month }}\end{array}$} & \multirow[t]{2}{*}{$\begin{array}{c}\text { th } \\
\text { month }\end{array}$} & \multirow[t]{2}{*}{$\begin{array}{c}12^{\text {th }} \\
\text { month }\end{array}$} & \multirow[t]{2}{*}{ Mean } \\
\hline & 1 day & 5 day & 1 day & 5 day & 1 day & 5 day & & & & \\
\hline 2012 T 73 & 13.31 & 11.46 & 12.68 & 11.54 & 11.30 & 10.86 & 13.9 & 9.0 & 3.9 & 8.9 \\
\hline 2012 T 81 & 12.70 & 11.92 & 11.93 & 12.04 & 11.09 & 10.16 & 6.1 & -0.9 & 8.4 & 4.5 \\
\hline 2012 T 106 & 12.54 & 11.00 & 11.96 & 11.85 & 12.35 & 11.56 & 12.3 & 0.9 & 6.4 & 6.5 \\
\hline 2012 T 180 & 12.78 & 11.42 & 12.42 & 10.79 & 11.16 & 11.82 & 10.6 & 13.1 & -5.9 & 6.0 \\
\hline 2003 V 46 ( & 13.64 & 11.69 & 12.53 & 12.42 & 12.22 & 10.46 & 14.3 & 0.9 & 14.4 & 9.9 \\
\hline Midlate & & & & & $\begin{array}{r}1 \\
\mathrm{mo}\end{array}$ & & $\begin{array}{c}12^{\text {th }} \\
\text { month }\end{array}$ & $\begin{array}{l}13^{\text {th }} \\
\text { month }\end{array}$ & $\begin{array}{c}14^{\text {th }} \\
\text { month }\end{array}$ & Mean \\
\hline 2012 T 58 & 13.27 & 10.62 & 12.45 & 11.11 & 12.84 & 10.68 & 20.0 & 10.8 & 16.8 & 15.9 \\
\hline 2012 T 72 & 12.38 & 9.60 & 11.74 & 11.60 & 12.43 & 9.62 & 22.5 & 1.2 & 22.6 & 15.4 \\
\hline 2012 T 78 & 13.12 & 11.41 & 12.73 & 10.56 & 12.03 & 10.52 & 13.0 & 17.0 & 12.6 & 14.2 \\
\hline 2012 T 182 & 9.90 & 10.32 & 12.95 & 10.61 & 11.13 & 9.92 & -4.2 & 18.1 & 10.9 & 8.2 \\
\hline CO 99004 & 13.76 & 12.04 & 12.49 & 10.66 & 13.76 & 12.10 & 12.5 & 14.7 & 12.1 & 13.1 \\
\hline
\end{tabular}

\section{Effect of delayed harvesting on CCS} percent

The CCS percent was significantly affected by varieties, months as well as with their interaction. Among the early clones, the CCS percent has been decreased from $10^{\text {th }}$ month to 12 month harvesting (Table 7). Less reduction from $10^{\text {th }}$ month to $11^{\text {th }}$ month was noticed with 2012 T 106 (-0.83\%) followed by $2012 \mathrm{~T}$ 73 and 2012 T 180. The clone 2012 T 106 showed less reduction $(1.41 \%)$ from $10^{\text {th }}$ to $12^{\text {th }}$ month harvesting followed by $2012 \mathrm{~T} 180$ and $2012 \mathrm{~T} 73$.

The midlate clones did not showed any particulars trend in increase / decrease of CCS. The clone 2012 T 182 showed increase in CCS percent from $12^{\text {th }}$ to $13^{\text {th }}$ month (5.04\%).

The CCS percent has been decreased at $13^{\text {th }}$ to $14^{\text {th }}$ month harvesting when compared with $12^{\text {th }}$ month harvesting (normal time) in $2012 \mathrm{~T}$ 58. Similar results were reported by Indrajith and Natarajan (2011) and Charumathi et al., (2009) for promising sugarcane clones

\section{Effect of delayed crushing on CCS percent}

Varieties and staling period showed significant influence on CCS percent. However their interaction was not significant. When compared check variety all early clones recorded less reduction in CCS percent at $10^{\text {th }}$ and $12^{\text {th }}$ month harvesting. Among early clones $2012 \mathrm{~T} 81$ showed less reduction in the mean CCS percent from $1^{\text {st }}$ day to $5^{\text {th }}$ day (4.5\%) followed by $2012 \mathrm{~T} 106$ (6.5\%). Midlate clones showed more reduction in CCS percent from $1^{\text {st }}$ day to $5^{\text {th }}$ day. $2012 \mathrm{~T} 182$ showed less reduction in mean CCS percent (9.9\%) than other clones. The CCS\% has decreased from $1^{\text {st }}$ day to $5^{\text {th }}$ day in all early and midlate clones (Reddy and Nagamadhuri, 2014) (Table 8).

From this study it was concluded that the early clones $2012 \mathrm{~T} 81$ was suitable for $11^{\text {th }}$ month harvesting, $2012 \mathrm{~T} 180$ was suitable for $12^{\text {th }}$ month harvesting. The midlate clones $2012 \mathrm{~T}$ 72 and $2012 \mathrm{~T} 182$ were suitable for $13^{\text {th }}$ month harvesting, 2012 T 78 was suitable for $14^{\text {th }}$ month harvesting. The clones $2012 \mathrm{~T} 81$ 
(Early) and 2012 T 182 (Midlate) showed tolerance for post harvest deterioration.

\section{References}

Alexander, A.G., 1973. Evaluation of sugarenzyme relationships among twelve sugarcane varieties. Puerto Rice Journal of Agricultural University. 51(1): 29-38.

Charumathi, M., Naidu, N.V., and Ravi Kumar., $\quad$ B.N.V.S.R. 2009. Performance of early maturing clones in zonal varietal trails. Proceedings of $40^{\text {th }}$ Annual convention of SISSTA. 39-40.

Gupta, A.P., Shukla, S.P. and Juneja, I.S., 1975. Cane varieties v/s deterioration after harvest, STIA, Golden jubilee. $39-42$.

Indrajith, J., and Natarajan, S., 2011. Performance of CoV 92102-An elite sugarcane cultivar in deltaic region of Tamilnadu. Indian Sugar. 49-50.

Meade, G.P., and Chen, J.P.G., 1977. Cane sugar handbook, $10^{\text {th }}$ edition. A Wiley Inter Science Publication, Johb Wiley and Sons, New York.

Panse, V.G., and Sukhatme, P.V., 1985.
Statistical methods for agricultural workers. ICAR, New Delhi, p.232.

Reddy, Y.S., and Nagamadhuri, K.V., 2014. Impact of delayed crushing on the Bioscan 9(2): 519-523.

Sharma, K. P., and Sunita, S., 1994. Post harvest loss in sugarcane on staling. National symposium on improvement in sugarcane quality fo increasing suga production, Indian Institute of Sugarcane Research, Lucknow, India. 19-22 September. Pp: 21-23.

Siddhant, Srivastava, R.P., Singh S.B. and Sharma, M.L., 2008. Assessment of sugar losses during staling in different varieties of sugarcane under subtropical condition. Sugar Tech. 10(4): 350-354.

Solomon, S., Shrivastava, A.K., Srivastava, B.L. and Madan, V.K., 1997. Premilling sugarcane losses and their management in sugarcane. Technical Bulletin, No.37. Indian Institute of Sugarcane Research, Lucknow. Pp. 1127.

Solomon, S. 2009. Post harvest deterioration of sugarcane. Sugar Tech 11(2): 109123.

\section{How to cite this article:}

Vajantha, B., M. Hemanth Kumar, K.R. Tagore, N.V. Sarala and Hemalatha, T.M. 2019. Effect of Delayed Harvesting and Crushing on Yield and Juice Quality of Promising Sugarcane Clones. Int.J.Curr.Microbiol.App.Sci. 8(11): 1745-1754. doi: https://doi.org/10.20546/ijcmas.2019.811.204 